\author{
BENHARRAT BELAÏDI
}

\title{
GROWTH OF MEROMORPHIC SOLUTIONS OF FINITE LOGARITHMIC ORDER OF LINEAR DIFFERENCE EQUATIONS
}

Abstract. In this paper, we deal with the growth and the oscillation of solutions of the linear difference equation

$$
\begin{aligned}
a_{n}(z) f(z+n)+a_{n-1}(z) f(z+n-1) \\
+\cdots+a_{1}(z) f(z+1)+a_{0}(z) f(z)=0
\end{aligned}
$$

where $a_{n}(z), \cdots, a_{0}(z)$ are meromorphic functions of finite logarithmic order such that $a_{n}(z) a_{0}(z) \not \equiv 0$.

KEY WORDS: linear difference equations, meromorphic function, logarithmic order, logarithmic type.

AMS Mathematics Subject Classification: 39A10, 30D35, 39A12.

\section{Introduction and main results}

Throughout this paper, we assume that the reader is familiar with the fundamental results and the standard notations of the Nevanlinna theory of meromorphic functions ([6], [12]). Recently, many articles focused on complex difference equations. The back-ground for these studies lies in the recent difference counterparts of Nevanlinna theory. The key result here is the difference analogue of the lemma on the logarithmic derivative obtained by Halburd-Korhonen [4,5] and Chiang-Feng [3], independently.

Definition $1([6])$. Let $f$ be a meromorphic function of order $\rho(f)=\rho$ $(0<\rho<\infty)$, the type of $f$ is defined as

$$
\tau(f)=\limsup _{r \rightarrow+\infty} \frac{T(r, f)}{r^{\rho}} .
$$

If $f$ is an entire function of order $\rho(0<\rho<\infty)$, we can define the $M$-type by

$$
\tau_{M}(f)=\limsup _{r \rightarrow+\infty} \frac{\log M(r, f)}{r^{\rho}} .
$$


Remark 1. We have not always the equality $\tau_{M}(f)=\tau(f)$, for example $\tau\left(e^{z}\right)=\frac{1}{\pi}<1=\tau_{M}\left(e^{z}\right)$. By $T(r, f) \leq \log ^{+} M(r, f)$, it's easy to obtain the following

$$
\tau(f) \leq \tau_{M}(f) .
$$

Definition $2([12])$. Let $f$ be a meromorphic function. Then the exponent of convergence of the sequence of zeros and distinct zeros of $f(z)$ are defined respectively by

$$
\begin{aligned}
& \lambda(f)=\limsup _{r \rightarrow+\infty} \frac{\log N\left(r, \frac{1}{f}\right)}{\log r}, \\
& \bar{\lambda}(f)=\limsup _{r \rightarrow+\infty} \frac{\log \bar{N}\left(r, \frac{1}{f}\right)}{\log r},
\end{aligned}
$$

where $N\left(r, \frac{1}{f}\right)\left(\bar{N}\left(r, \frac{1}{f}\right)\right)$ is the integrated counting function of zeros (distinct zeros) of $f(z)$ in $\{z:|z| \leq r\}$.

In recent paper [3], Chiang and Feng investigated meromorphic solutions of the linear difference equation

$$
\begin{aligned}
& a_{n}(z) f(z+n)+a_{n-1}(z) f(z+n-1) \\
& \quad+\cdots+a_{1}(z) f(z+1)+a_{0}(z) f(z)=0,
\end{aligned}
$$

where $a_{n}(z), \cdots, a_{0}(z)$ are entire functions such that $a_{n}(z) a_{0}(z) \not \equiv 0$, and proved the following result.

Theorem A ([3]) . Let $a_{0}(z), a_{1}(z), \cdots, a_{n}(z)$ be entire functions such that there exists an integer $l, 0 \leq l \leq n$ such that

$$
\rho\left(a_{l}\right)>\max _{0 \leq j \leq n, j \neq l}\left\{\rho\left(a_{j}\right)\right\} .
$$

If $f(z)$ is a meromorphic solution of $(1)$, then $\rho(f) \geq \rho\left(a_{l}\right)+1$.

Note that in Theorem A, equation (1) has only one dominating coefficient $a_{l}$. For the case when there is more than one of coefficients which have the maximal order, Laine and Yang [9] obtained the following result.

Theorem B ([9]). Let $a_{0}(z), a_{1}(z), \cdots, a_{n}(z)$ be entire functions of $f_{i-}$ nite order such that among those having the maximal order $\rho=\max _{0 \leq j \leq n}\left\{\rho\left(a_{j}\right)\right\}$, one has exactly its type strictly greater than the others. Then for any meromorphic solution of (1), we have $\rho(f) \geq \rho+1$.

Obviously, we have $\rho\left(a_{l}\right)>0$ and $\rho>0$ in Theorems A-B. Thus, a natural problem which arises: How to express the growth of solutions of 
(1) when all coefficients $a_{0}(z), a_{1}(z), \cdots, a_{n}(z)$ are meromorphic functions and of order zero in $\mathbb{C}$. The main purpose of this paper is to adopt the idea of finite logarithmic order due to Chern [2] to extend some results obtained recently by the author and Latreuch in [10] for meromorphic solutions to equation (1) of zero order in $\mathbb{C}$.

Definition 3 ([2]). The logarithmic order of a meromorphic function $f$ is defined as

$$
\rho_{\log }(f)=\limsup _{r \rightarrow+\infty} \frac{\log T(r, f)}{\log \log r} .
$$

If $f$ is an entire function, then

$$
\rho_{\log }(f)=\limsup _{r \rightarrow+\infty} \frac{\log T(r, f)}{\log \log r}=\limsup _{r \rightarrow+\infty} \frac{\log \log M(r, f)}{\log \log r} .
$$

Remark 2. It is evident that the logarithmic order of any non-constant rational function $f$ is one, and thus, any transcendental meromorphic function in the plane has logarithmic order no less than one. However, a function of logarithmic order one is not necessarily a rational function. Constant functions have zero logarithmic order, while there are no meromorphic functions of logarithmic order between zero and one. Moreover, any meromorphic function with finite logarithmic order in the plane is of order zero.

Definition 4 ([1]). The logarithmic type of a meromorphic function $f$ with $1 \leq \rho_{\log }(f)<+\infty$ is defined by

$$
\tau_{\log }(f)=\limsup _{r \rightarrow+\infty} \frac{T(r, f)}{(\log r)^{\rho_{\log }(f)}} .
$$

If $f$ is an entire function with $1 \leq \rho_{\log }(f)<+\infty$, then

$$
\tau_{\log }(f)=\limsup _{r \rightarrow+\infty} \frac{T(r, f)}{(\log r)^{\rho_{\log }(f)}}=\limsup _{r \rightarrow+\infty} \frac{\log M(r, f)}{(\log r)^{\rho_{\log }(f)}} .
$$

Remark 3. It is evident that the logarithmic type of any non-constant polynomial $Q$ equals its degree $\operatorname{deg}(Q)$; that any non-constant rational function is of finite logarithmic type, and that any transcendental meromorphic function whose logarithmic order equals one in the plane must be of infinite logarithmic type.

Definition $5([1],[2])$. The logarithmic exponent of convergence of a-points of a meromorphic function $f$ is equal to the logarithmic order of $n(r, f=a)$ which is defined as

$$
\lambda_{\log }(f, a)=\limsup _{r \rightarrow+\infty} \frac{\log n\left(r, \frac{1}{f-a}\right)}{\log \log r} .
$$


The logarithmic exponent of convergence of zeros or distinct zeros of $f$ are defined by

$$
\lambda_{\log }(f)=\lambda_{\log }(f, 0)=\limsup _{r \rightarrow+\infty} \frac{\log n\left(r, \frac{1}{f}\right)}{\log \log r}
$$

or

$$
\bar{\lambda}_{\log }(f)=\bar{\lambda}_{\log }(f, 0)=\limsup _{r \rightarrow+\infty} \frac{\log \bar{n}\left(r, \frac{1}{f}\right)}{\log \log r} .
$$

respectively, where $n\left(r, \frac{1}{f}\right)$ (or $\left.\bar{n}\left(r, \frac{1}{f}\right)\right)$ denotes the number of zeros (or distinct zeros) of $f$ in the disc $|z| \leq r$.

Remark 4. It is trivial that $\lambda(f)$ (or $\bar{\lambda}(f))$ ) can also be given by making use of the notation of the counting function of zeros (or distinct zeros) of $f, N\left(r, \frac{1}{f}\right)$ (or $\bar{N}\left(r, \frac{1}{f}\right)$, to replace the notations $n\left(r, \frac{1}{f}\right)\left(\right.$ or $\left.\bar{n}\left(r, \frac{1}{f}\right)\right)$, respectively (see e.g. [12], Theorem 2.1). However, it does not hold for the case of logarithmic order. The logarithmic order of $N\left(r, \frac{1}{f}\right)$ is equals $\lambda_{\log }(f)+1$, (see [2], Theorem 4.1).

Recently, the concept of logarithmic order has been used to investigate the growth and the oscillation of solutions of linear differential equations in the complex plane [1] and complex linear difference and $q$-difference equations in the complex plane and in the unit disc $([7],[8],[11])$. In this paper, we obtain the following results.

Theorem 1. Let $a_{0}(z), a_{1}(z), \cdots, a_{n}(z)$ be meromorphic functions such that there exists coefficient $a_{l}$ satisfying $\lambda_{\log }\left(\frac{1}{a_{l}}\right)<\rho_{\log }\left(a_{l}\right)=\rho$ $(1 \leq \rho<\infty)$. Suppose that

$$
\max \left\{\rho_{\log }\left(a_{j}\right): 0 \leq j \leq n, j \neq l\right\}<\rho_{\log }\left(a_{l}\right) .
$$

If $f(z)$ is a meromorphic solution of $(1)$, then $\rho_{\log }(f) \geq \rho_{\log }\left(a_{l}\right)+1$.

Theorem 2. Let $a_{0}(z), a_{1}(z), \cdots, a_{n}(z)$ be meromorphic functions such that there exists coefficient $a_{l}$ satisfying $\lambda_{\log }\left(\frac{1}{a_{l}}\right)<\rho_{\log }\left(a_{l}\right)=\rho$ $(1 \leq \rho<\infty), \tau_{\log }\left(a_{l}\right)=\tau(0<\tau<\infty)$. Suppose that

$$
\max \left\{\rho_{\log }\left(a_{j}\right): 0 \leq j \leq n, j \neq l\right\} \leq \rho
$$

and

$$
\sum_{\substack{\log \left(a_{j}\right)=\rho_{\log }\left(a_{l}\right) \\ j \neq l}} \tau_{\log }\left(a_{j}\right)<\tau .
$$

If $f(z)$ is a meromorphic solution of $(1)$, then $\rho_{\log }(f) \geq \rho_{\log }\left(a_{l}\right)+1$. 
Remark 5. If $\rho_{\log }\left(a_{j}\right)<\rho_{\log }\left(a_{l}\right)$ for all $j=0,1, \cdots, l-1, l+1, \cdots, n$, then the sum in (4) is empty, and Theorem 2 reduces to Theorem 1.

Theorem 3. Let $a_{0}(z), a_{1}(z), \cdots, a_{n}(z), F(z)(\not \equiv 0)$ be finite logarithmic order meromorphic functions. If $f$ is a meromorphic solution of the equation

$$
a_{n} f(z+n)+a_{n-1} f(z+n-1)+\cdots+a_{1} f(z+1)+a_{0} f(z)=F
$$

with

$$
\max \left\{\rho_{\log }\left(a_{j}\right) \quad(j=0, \cdots, n), \rho_{\log }(F)\right\}<\rho_{\log }(f),
$$

then $\rho_{\log }(f)=\lambda_{\log }(f)+1$.

Theorem 4. Under the assumptions of Theorem 2, let $\varphi$ be a meromorphic function such that one of the following conditions holds:

(i) $\varphi$ is not a solution of (1) with $\rho_{\log }(\varphi)<\rho_{\log }(f)$;

(ii) $\varphi \not \equiv 0$ and $\rho_{\log }(\varphi)<\rho_{\log }\left(a_{l}\right)+1$.

Then $\lambda_{\log }(f-\varphi)+1=\rho_{\log }(f)$.

Corollary. Under the assumptions of Theorem 4, we have $\lambda_{\log }(f-z)+$ $1=\rho_{\log }(f)$.

\section{Some lemmas}

We need the following lemmas to prove our results.

Lemma 1. Let $f$ be a meromorphic function with $\rho_{\log }(f)=\rho \geq 1$. Then there exists a set $E_{1} \subset[1,+\infty)$ with infinite logarithmic measure such that

$$
\lim _{\substack{r \rightarrow \infty \\ r \in E_{1}}} \frac{\log T(r, f)}{\log \log r}=\rho .
$$

Proof. Since $\rho_{\log }(f)=\rho$, then there exists a sequence $\left\{r_{n}\right\}_{n=1}^{\infty}$ tending to $\infty$ satisfying $\left(1+\frac{1}{n}\right) r_{n}<r_{n+1}$ and

$$
\lim _{r_{n} \rightarrow \infty} \frac{\log T\left(r_{n}, f\right)}{\log \log r_{n}}=\rho_{\log }(f) .
$$

So, there exists an integer $n_{1}$ such that for all $n \geq n_{1}$, for any $r \in\left[r_{n},\left(1+\frac{1}{n}\right) r_{n}\right]$, we have

$$
\frac{\log T\left(r_{n}, f\right)}{\log \log \left(1+\frac{1}{n}\right) r_{n}} \leq \frac{\log T(r, f)}{\log \log r} \leq \frac{\log T\left(\left(1+\frac{1}{n}\right) r_{n}, f\right)}{\log \log r_{n}}
$$


Set $E_{1}=\bigcup_{n=n_{1}}^{\infty}\left[r_{n},\left(1+\frac{1}{n}\right) r_{n}\right]$, we obtain

$$
\lim _{\substack{r \rightarrow \infty \\ r \in E_{1}}} \frac{\log T(r, f)}{\log \log r}=\lim _{r_{n} \rightarrow \infty} \frac{\log T\left(r_{n}, f\right)}{\log \log r_{n}}
$$

and $\operatorname{lm}\left(E_{1}\right)=\sum_{n=n_{1}}^{\infty} \int_{r_{n}}^{\left(1+\frac{1}{n}\right) r_{n}} \frac{d t}{t}=\sum_{n=n_{1}}^{\infty} \log \left(1+\frac{1}{n}\right)=\infty$. Thus, the proof of the lemma is completed.

Lemma 2. Let $f$ be a meromorphic function with finite logarithmic order $1 \leq \rho_{\log }(f)<+\infty$ and finite logarithmic type $0<\tau_{\log }(f)<+\infty$. Then for any given $\beta<\tau_{\log }(f)$ there exists a subset $E_{2}$ of $[1,+\infty)$ that has infinite logarithmic measure such that $T(r, f)>\beta(\log r)^{\rho_{\log }(f)}$, holds for all $r \in E_{2}$.

Proof. By Definition 4, there exists an increasing sequence $\left\{r_{m}\right\}\left(r_{m} \rightarrow\right.$ $+\infty)$ satisfying $\left(1+\frac{1}{m}\right) r_{m}<r_{m+1}$ and

$$
\lim _{m \rightarrow+\infty} \frac{T\left(r_{m}, f\right)}{\left(\log r_{m}\right)^{\rho_{\log }(f)}}=\tau_{\log }(f) .
$$

So, there exists a positive integer $m_{0}$ such that for all $m \geq m_{0}$ and for any given $0<\varepsilon<\tau_{\log }(f)-\beta$, we have

$$
T\left(r_{m}, f\right)>\left(\tau_{\log }(f)-\varepsilon\right)\left(\log r_{m}\right)^{\rho_{\log }(f)} .
$$

Since

$$
\lim _{m \rightarrow+\infty}\left(\frac{\log \frac{m}{m+1} r}{\log r}\right)^{\rho_{\log }(f)}=1
$$

then for any given $\beta<\tau_{\log }(f)-\varepsilon$, there exists a positive integer $m_{1}$ such that for all $m \geq m_{1}$ we have

$$
\left(\frac{\log \frac{m}{m+1} r}{\log r}\right)^{\rho_{\log }(f)}>\frac{\beta}{\tau_{\log }(f)-\varepsilon}
$$

Take $m \geq m_{2}=\max \left\{m_{1}, m_{0}\right\}$. By (6) and (7), for any $r \in\left[r_{m},\left(1+\frac{1}{m}\right) r_{m}\right]$

$$
\begin{aligned}
T(r, f) & \geq T\left(r_{m}, f\right)>\left(\tau_{\log }(f)-\varepsilon\right)\left(\log r_{m}\right)^{\rho_{\log }(f)} \\
& \geq\left(\tau_{\log }(f)-\varepsilon\right)\left(\log \frac{m}{m+1} r\right)^{\rho_{\log }(f)}>\beta(\log r)^{\rho_{\log }(f)}
\end{aligned}
$$


Set $E_{2}=\bigcup_{m=m_{2}}^{+\infty}\left[r_{m},\left(1+\frac{1}{m}\right) r_{m}\right]$. Then there holds

$$
\operatorname{lm}\left(E_{2}\right)=\sum_{m=m_{2}}^{+\infty} \int_{r_{m}}^{\left(1+\frac{1}{m}\right) r_{m}} \frac{d t}{t}=\sum_{m=m_{2}}^{+\infty} \log \left(1+\frac{1}{m}\right)=+\infty .
$$

Lemma 3. Let $f_{1}, f_{2}$ be meromorphic functions satisfying $\rho_{\log }\left(f_{1}\right)>$ $\rho_{\log }\left(f_{2}\right)$. Then there exists a set $E_{3} \subset(1,+\infty)$ having infinite logarithmic measure such that for all $r \in E_{3}$, we have

$$
\lim _{r \rightarrow \infty} \frac{T\left(r, f_{2}\right)}{T\left(r, f_{1}\right)}=0 .
$$

Proof. Set $\rho_{1}=\rho_{\log }\left(f_{1}\right), \rho_{2}=\rho_{\log }\left(f_{2}\right)\left(\rho_{1}>\rho_{2}\right)$. By Lemma 1 , there exists a set $E_{3} \subset(1,+\infty)$ having infinite logarithmic measure such that for any given $0<\varepsilon<\frac{\rho_{1}-\rho_{2}}{2}$ and all sufficiently large $r \in E_{3}$

$$
T\left(r, f_{1}\right)>(\log r)^{\rho_{1}-\varepsilon}
$$

and for all sufficiently large $r$, we have

$$
T\left(r, f_{2}\right)<(\log r)^{\rho_{2}+\varepsilon} .
$$

From this we can get

$$
\frac{T\left(r, f_{2}\right)}{T\left(r, f_{1}\right)}<\frac{(\log r)^{\rho_{2}+\varepsilon}}{(\log r)^{\rho_{1}-\varepsilon}}=\frac{1}{(\log r)^{\rho_{1}-\rho_{2}-2 \varepsilon}}, \quad r \in E_{3} .
$$

Since $0<\varepsilon<\frac{\rho_{1}-\rho_{2}}{2}$, then we obtain

$$
\lim _{r \rightarrow \infty} \frac{T\left(r, f_{2}\right)}{T\left(r, f_{1}\right)}=0, \quad r \in E_{3} .
$$

Lemma 4 ([3]). Let $\alpha, R, R^{\prime}$ be real numbers such that $0<\alpha<1, R>0$, and let $\eta$ be a non-zero complex number. Then there is a positive constant $C_{\alpha}$ depending only on $\alpha$ such that for a given meromorphic function $f(z)$ we have, when $|z|=r, \max \{1, r+|\eta|\}<R<R^{\prime}$, the estimate

$$
\begin{aligned}
& m\left(r, \frac{f(z+\eta)}{f(z)}\right)+m\left(r, \frac{f(z)}{f(z+\eta)}\right) \\
& \leq \frac{2|\eta| R}{(R-r-|\eta|)^{2}}\left(m(R, f)+m\left(R, \frac{1}{f}\right)\right) \\
& \quad+\frac{2 R^{\prime}}{\left(R^{\prime}-R\right)}\left(\frac{|\eta|}{R-r-|\eta|}+\frac{C_{\alpha}|\eta|^{\alpha}}{(1-\alpha) r^{\alpha}}\right)\left(N\left(R^{\prime}, f\right)+N\left(R^{\prime}, \frac{1}{f}\right)\right) .
\end{aligned}
$$


Remark 6. We note that the estimate (8) follows from the proof of Theorem 2.4 in [3].

Lemma 5. Let $\eta_{1}, \eta_{2}$ be two arbitrary complex numbers such that $\eta_{1} \neq \eta_{2}$ and let $f(z)$ be a finite logarithmic order meromorphic function. Let $\rho$ be the logarithmic order of $f(z)$. Then for each $\varepsilon>0$, we have

$$
m\left(r, \frac{f\left(z+\eta_{1}\right)}{f\left(z+\eta_{2}\right)}\right)=O\left((\log r)^{\rho-1+\varepsilon}\right) .
$$

Proof. We have

$$
\begin{aligned}
m\left(r, \frac{f\left(z+\eta_{1}\right)}{f\left(z+\eta_{2}\right)}\right) \leq & m\left(r, \frac{f\left(z+\eta_{1}\right)}{f(z)}\right)+m\left(r, \frac{f(z)}{f\left(z+\eta_{2}\right)}\right) \\
\leq & m\left(r, \frac{f\left(z+\eta_{1}\right)}{f(z)}\right)+m\left(r, \frac{f(z)}{f\left(z+\eta_{1}\right)}\right) \\
& +m\left(r, \frac{f(z)}{f\left(z+\eta_{2}\right)}\right)+m\left(r, \frac{f\left(z+\eta_{2}\right)}{f(z)}\right) .
\end{aligned}
$$

Since $f(z)$ has finite logarithmic order $\rho_{\log }(f)=\rho<+\infty$, so given $\varepsilon$, $0<\varepsilon<2$, we have

$$
T(r, f)=O\left((\log r)^{\rho+\frac{\varepsilon}{2}}\right)
$$

for all $r$. By using (8), we obtain from (10)

$$
\begin{aligned}
m(r & \left.\frac{f\left(z+\eta_{1}\right)}{f\left(z+\eta_{2}\right)}\right) \leq \frac{2\left|\eta_{1}\right| R}{\left(R-r-\left|\eta_{1}\right|\right)^{2}}\left(m(R, f)+m\left(R, \frac{1}{f}\right)\right) \\
& +\frac{2 R^{\prime}}{\left(R^{\prime}-R\right)}\left(\frac{\left|\eta_{1}\right|}{R-r-\left|\eta_{1}\right|}+\frac{C_{\alpha}\left|\eta_{1}\right|^{\alpha}}{(1-\alpha) r^{\alpha}}\right)\left(N\left(R^{\prime}, f\right)+N\left(R^{\prime}, \frac{1}{f}\right)\right) \\
& +\frac{2\left|\eta_{2}\right| R}{\left(R-r-\left|\eta_{2}\right|\right)^{2}}\left(m(R, f)+m\left(R, \frac{1}{f}\right)\right) \\
& +\frac{2 R^{\prime}}{\left(R^{\prime}-R\right)}\left(\frac{\left|\eta_{2}\right|}{R-r-\left|\eta_{2}\right|}+\frac{C_{\alpha}\left|\eta_{2}\right|^{\alpha}}{(1-\alpha) r^{\alpha}}\right)\left(N\left(R^{\prime}, f\right)+N\left(R^{\prime}, \frac{1}{f}\right)\right) \\
= & \left(\frac{2\left|\eta_{1}\right| R}{\left(R-r-\left|\eta_{1}\right|\right)^{2}}+\frac{2\left|\eta_{2}\right| R}{\left(R-r-\left|\eta_{2}\right|\right)^{2}}\right)\left(m(R, f)+m\left(R, \frac{1}{f}\right)\right) \\
& +\frac{2 R^{\prime}}{\left(R^{\prime}-R\right)}\left(\frac{\left|\eta_{1}\right|}{R-r-\left|\eta_{1}\right|}+\frac{C_{\alpha}\left|\eta_{1}\right|^{\alpha}}{(1-\alpha) r^{\alpha}}\right. \\
& \left.+\frac{\left|\eta_{2}\right|}{R-r-\left|\eta_{2}\right|}+\frac{C_{\alpha}\left|\eta_{2}\right|^{\alpha}}{(1-\alpha) r^{\alpha}}\right)\left(N\left(R^{\prime}, f\right)+N\left(R^{\prime}, \frac{1}{f}\right)\right) .
\end{aligned}
$$

By choosing $\alpha=1-\frac{\varepsilon}{2}, R=2 r, R^{\prime}=3 r$ and $r>\max \left\{\left|\eta_{1}\right|,\left|\eta_{2}\right|, 1 / 2\right\}$ in (12), we get

$$
m\left(r, \frac{f\left(z+\eta_{1}\right)}{f\left(z+\eta_{2}\right)}\right) \leq\left(\frac{4\left|\eta_{1}\right| r}{\left(r-\left|\eta_{1}\right|\right)^{2}}+\frac{4\left|\eta_{2}\right| r}{\left(r-\left|\eta_{2}\right|\right)^{2}}\right)\left(m(2 r, f)+m\left(2 r, \frac{1}{f}\right)\right)
$$




$$
\begin{aligned}
& +6\left(\frac{\left|\eta_{1}\right|}{r-\left|\eta_{1}\right|}+\frac{2 C_{\alpha}\left|\eta_{1}\right|^{1-\frac{\varepsilon}{2}}}{\varepsilon r^{1-\frac{\varepsilon}{2}}}+\frac{\left|\eta_{2}\right|}{r-\left|\eta_{2}\right|}+\frac{2 C_{\alpha}\left|\eta_{2}\right|^{1-\frac{\varepsilon}{2}}}{\varepsilon r^{1-\frac{\varepsilon}{2}}}\right) \\
& \times\left(N(3 r, f)+N\left(3 r, \frac{1}{f}\right)\right) \\
\leq & 4\left[\frac{4\left|\eta_{1}\right| r}{\left(r-\left|\eta_{1}\right|\right)^{2}}+\frac{4\left|\eta_{2}\right| r}{\left(r-\left|\eta_{2}\right|\right)^{2}}\right. \\
& \left.+6\left(\frac{\left|\eta_{1}\right|}{r-\left|\eta_{1}\right|}+\frac{\left|\eta_{2}\right|}{r-\left|\eta_{2}\right|}+\frac{2 C_{\alpha}\left(\left|\eta_{1}\right|^{1-\frac{\varepsilon}{2}}+\left|\eta_{2}\right|^{1-\frac{\varepsilon}{2}}\right)}{\varepsilon r^{1-\frac{\varepsilon}{2}}}\right)\right] T(3 r, f) .
\end{aligned}
$$

By using the estimate (11), we have

$$
\begin{aligned}
& m\left(r, \frac{f\left(z+\eta_{1}\right)}{f\left(z+\eta_{2}\right)}\right) \leq 4 K\left[\frac{4 \mid \eta_{1} \| r}{\left(r-\left|\eta_{1}\right|\right)^{2}}+\frac{4\left|\eta_{2}\right| r}{\left(r-\left|\eta_{2}\right|\right)^{2}}\right. \\
& \left.\quad+6\left(\frac{\left|\eta_{1}\right|}{r-\left|\eta_{1}\right|}+\frac{\left|\eta_{2}\right|}{r-\left|\eta_{2}\right|}+\frac{2 C_{\alpha}\left(\left|\eta_{1}\right|^{1-\frac{\varepsilon}{2}}+\left|\eta_{2}\right|^{1-\frac{\varepsilon}{2}}\right)}{\varepsilon r^{1-\frac{\varepsilon}{2}}}\right)\right](\log 3 r)^{\rho+\frac{\varepsilon}{2}} \\
& \quad \leq M(\log r)^{\rho+\varepsilon-1},
\end{aligned}
$$

where $K>0, M>0$ are some constants. This completes the proof.

Lemma 6 ([2]). Let $f$ be a meromorphic function of finite logarithmic order, and let $a \in \overline{\mathbb{C}}$. Then the logarithmic order of $n(r, 1 /(f-a))$ equals to $\lambda_{\log }(f, a)$. Moreover, $N(r, 1 /(f-a))$ is of logarithmic order $\lambda_{\log }(f, a)+1$.

Remark 7. We point out that the first assertion of Lemma 6 is Theorem 3.1 in [2], while the second one is in Theorem 4.1 of the same paper.

Lemma 7. Let $f$ and $g$ be non-constant meromorphic functions of logarithmic order. Then we have

$$
\rho_{\log }(f+g) \leq \max \left\{\rho_{\log }(f), \rho_{\log }(g)\right\}
$$

and

$$
\rho_{\log }(f g) \leq \max \left\{\rho_{\log }(f), \rho_{\log }(g)\right\} .
$$

Furthermore, if $\rho_{\log }(f)>\rho_{\log }(g)$, then we obtain

$$
\rho_{\log }(f+g)=\rho_{\log }(f g)=\rho_{\log }(f) .
$$

Proof. Set $\rho_{\log }(f)=\rho_{1}$ and $\rho_{\log }(g)=\rho_{2}$. For any given $\varepsilon>0$, we have

$$
\begin{aligned}
T(r, f+g) & \leq T(r, f)+T(r, g)+O(1) \\
& \leq(\log r)^{\rho_{1}+\varepsilon}+(\log r)^{\rho_{2}+\varepsilon}+O(1) \\
& \leq 2(\log r)^{\max \left\{\rho_{1}, \rho_{2}\right\}+\varepsilon}+O(1)
\end{aligned}
$$


and

$$
T(r, f g) \leq T(r, f)+T(r, g) \leq 2(\log r)^{\max \left\{\rho_{1}, \rho_{2}\right\}+\varepsilon}
$$

for all $r$ sufficiently large. Since $\varepsilon>0$ is arbitrary, from (13) and (14), we easily obtain

$$
\rho_{\log }(f+g) \leq \max \left\{\rho_{\log }(f), \rho_{\log }(g)\right\}
$$

and

$$
\rho_{\log }(f g) \leq \max \left\{\rho_{\log }(f), \rho_{\log }(g)\right\}
$$

Suppose now that $\rho_{\log }(f)>\rho_{\log }(g)$. Considering that

$$
T(r, f)=T(r, f+g-g) \leq T(r, f+g)+T(r, g)+O(1)
$$

and

$$
\begin{aligned}
T(r, f) & =T\left(r, \frac{f g}{g}\right) \leq T(r, f g)+T\left(r, \frac{1}{g}\right) \\
& =T(r, f g)+T(r, g)+O(1) .
\end{aligned}
$$

By (17) and (18), by the same method as above we obtain that

$$
\begin{gathered}
\rho_{\log }(f) \leq \max \left\{\rho_{\log }(f+g), \rho_{\log }(g)\right\}=\rho_{\log }(f+g), \\
\rho_{\log }(f) \leq \max \left\{\rho_{\log }(f g), \rho_{\log }(g)\right\}=\rho_{\log }(f g) .
\end{gathered}
$$

By using (15) and (19) we obtain $\rho_{\log }(f+g)=\rho_{\log }(f)$ and by $(16)$ and (20), we get $\rho_{\log }(f g)=\rho_{\log }(f)$.

Lemma 8. Let $f$ and $g$ be meromorphic functions in the complex plane such that $1 \leq \rho_{\log }(f), \rho_{\log }(g)<\infty$ and $0<\tau_{\log }(f), \tau_{\log }(g)<\infty$. Then we have

(i) If $\rho_{\log }(f)>\rho_{\log }(g)$, then we obtain

$$
\tau_{\log }(f+g)=\tau_{\log }(f g)=\tau_{\log }(f) .
$$

(ii) If $\rho_{\log }(f)=\rho_{\log }(g)$ and $\tau_{\log }(f) \neq \tau_{\log }(g)$, then we get

$$
\rho_{\log }(f+g)=\rho_{\log }(f g)=\rho_{\log }(f)=\rho_{\log }(g) .
$$


Proof. (i) Suppose that $\rho_{\log }(f)>\rho_{\log }(g)$. By using the definition of the type and since $\rho_{\log }(f+g)=\rho_{\log }(f)$, we get

$$
\begin{aligned}
\tau_{\log }(f+g)=\limsup _{r \rightarrow+\infty} \frac{T(r, f+g)}{(\log r)^{\rho_{\log }(f+g)}} \\
\quad \leq \limsup _{r \rightarrow+\infty} \frac{T(r, f)+T(r, g)+O(1)}{(\log r)^{\rho_{\log }(f)}} \\
\leq \limsup _{r \rightarrow+\infty} \frac{T(r, f)}{(\log r)^{\rho_{\log }(f)}}+\limsup _{r \rightarrow+\infty} \frac{T(r, g)+O(1)}{(\log r)^{\rho_{\log }(f)}} \\
=\limsup _{r \rightarrow+\infty} \frac{T(r, f)}{(\log r)^{\rho_{\log }(f)}}+\limsup _{r \rightarrow+\infty}\left(\frac{T(r, g)}{(\log r)^{\rho_{\log }(g)}} \frac{(\log r)^{\rho_{\log }(g)}}{(\log r)^{\rho_{\log }(f)}}\right) \\
\quad \leq \limsup _{r \rightarrow+\infty} \frac{T(r, f)}{(\log r)^{\rho_{\log }(f)}}+\limsup _{r \rightarrow+\infty} \frac{(\log r)^{\rho_{\log }(g)}}{(\log r)^{\rho_{\log }(f)}} \limsup _{r \rightarrow+\infty} \frac{T(r, g)}{(\log r)^{\rho_{\log }(g)}} \\
=\tau_{\log }(f) .
\end{aligned}
$$

Since $\rho_{\log }(f+g)=\rho_{\log }(f)>\rho_{\log }(g)$, then by (23), we obtain

$$
\tau_{\log }(f)=\tau_{\log }(f+g-g) \leq \tau_{\log }(f+g) .
$$

Hence $\tau_{\log }(f+g)=\tau_{\log }(f)$. By the same method as before, we have

$$
\begin{aligned}
\tau_{\log }(f g) & =\limsup _{r \rightarrow+\infty} \frac{T(r, f g)}{(\log r)^{\rho_{\log }(f g)}} \leq \limsup _{r \rightarrow+\infty} \frac{T(r, f)+T(r, g)}{(\log r)^{\rho_{\log }(f)}} \\
& \leq \limsup _{r \rightarrow+\infty} \frac{T(r, f)}{(\log r)^{\rho_{\log }(f)}}+\limsup _{r \rightarrow+\infty} \frac{T(r, g)}{(\log r)^{\rho_{\log }(f)}} \leq \tau_{\log }(f) .
\end{aligned}
$$

Since $\rho_{\log }(f g)=\rho_{\log }(f)>\rho_{\log }(g)=\rho_{\log }\left(\frac{1}{g}\right)$, then by (24), we obtain

$$
\tau_{\log }(f)=\tau_{\log }\left(f g \frac{1}{g}\right) \leq \tau_{\log }(f g) .
$$

Thus, $\tau_{\log }(f g)=\tau_{\log }(f)$.

(ii) Without loss of generality, we suppose that $\tau_{\log }(f)>\tau_{\log }(g)$. It's easy to see that

$$
\rho_{\log }(f+g) \leq \rho_{\log }(f)=\rho_{\log }(g) .
$$

If we suppose that $\rho_{\log }(f+g)<\rho_{\log }(f)=\rho_{\log }(g)$, then by $(21)$

$$
\tau_{\log }(g)=\tau_{\log }(f+g-f)=\tau_{\log }(f)
$$

which is a contradiction. Hence $\rho_{\log }(f+g)=\rho_{\log }(f)=\rho_{\log }(g)$. Also, we have

$$
\rho_{\log }(f g) \leq \rho_{\log }(f)=\rho_{\log }(g) \text {. }
$$


If we suppose $\rho_{\log }(f g)<\rho_{\log }(f)=\rho_{\log }\left(\frac{1}{f}\right)=\rho_{\log }(g)$, then by (21), we can write

$$
\tau_{\log }(g)=\tau_{\log }\left(f g \frac{1}{f}\right)=\tau_{\log }(f),
$$

which is a contradiction. Hence $\rho_{\log }(f g)=\rho_{\log }(f)=\rho_{\log }(g)$.

\section{Proof of the theorems and corollary}

Proof. of Theorem 1. If $\rho_{\log }(f)=\infty$, then the result is trivial. Next we suppose $\rho_{\log }(f)<\infty$. We divide through equation (1) by $f(z+l)$ to get

$$
\begin{aligned}
a_{l}(z)= & -\left(a_{n}(z) \frac{f(z+n)}{f(z+l)}+\cdots+a_{l-1}(z) \frac{f(z+l-1)}{f(z+l)}\right. \\
& +a_{l+1}(z) \frac{f(z+l+1)}{f(z+l)}+\cdots+a_{1}(z) \frac{f(z+1)}{f(z+l)} \\
& \left.+a_{0}(z) \frac{f(z)}{f(z+l)}\right) .
\end{aligned}
$$

It follows that

$$
\begin{aligned}
T\left(r, a_{l}\right) & =m\left(r, a_{l}\right)+N\left(r, a_{l}\right) \\
& \leq \sum_{\substack{j=0 \\
j \neq l}}^{n} m\left(r, a_{j}\right)+\sum_{\substack{j=0 \\
j \neq l}}^{n} m\left(r, \frac{f(z+j)}{f(z+l)}\right)+N\left(r, a_{l}\right)+O(1) \\
& \leq \sum_{\substack{j=0 \\
j \neq l}}^{n} T\left(r, a_{j}\right)+\sum_{\substack{j=0 \\
j \neq l}}^{n} m\left(r, \frac{f(z+j)}{f(z+l)}\right)+N\left(r, a_{l}\right)+O(1) .
\end{aligned}
$$

By Lemma 5, we have for sufficiently large $r$ and any given $\varepsilon>0$

$$
m\left(r, \frac{f(z+j)}{f(z+l)}\right)=O\left((\log r)^{\rho_{\log }(f)-1+\varepsilon}\right), \quad j=0, \cdots, n, \quad j \neq l .
$$

Let us choose $\sigma$ such that $\lambda_{\log }\left(\frac{1}{a_{l}}\right)<\sigma<\rho_{\log }\left(a_{l}\right)=\rho$. Then we have for any given $\varepsilon(0<\varepsilon<\rho-\sigma)$ and for sufficiently large $r$

$$
N\left(r, a_{l}\right) \leq(\log r)^{\lambda_{\log \left(\frac{1}{a_{l}}\right)+\varepsilon}} \leq(\log r)^{\sigma+\varepsilon} .
$$

Thus, by (27) and (28), we obtain from (26)

$$
T\left(r, a_{l}\right) \leq \sum_{\substack{j=0 \\ j \neq l}}^{n} T\left(r, a_{j}\right)+O\left((\log r)^{\rho_{\log }(f)-1+\varepsilon}\right)+(\log r)^{\sigma+\varepsilon} .
$$


Since $\max \left\{\rho_{\log }\left(a_{j}\right)(j=0, \cdots, n), j \neq l\right\}<\rho_{\log }\left(a_{l}\right)$, then by Lemma 3 , there exists a set $E_{3} \subset[1,+\infty)$ with infinite logarithmic measure such that

$$
\max \left\{\frac{T\left(r, a_{j}\right)}{T\left(r, a_{l}\right)}(j=0, \cdots, n), j \neq l\right\} \rightarrow 0, \quad r \rightarrow+\infty, \quad r \in E_{3} .
$$

Thus, by (29) and (30), we have for all $r \in E_{3}, r \rightarrow+\infty$

$$
(1-o(1)) T\left(r, a_{l}\right) \leq O\left((\log r)^{\rho_{\log }(f)-1+\varepsilon}\right)+(\log r)^{\sigma+\varepsilon} .
$$

Since $0<\varepsilon<\rho-\sigma$, then it follows from (31) that $\rho_{\log }(f) \geq \rho_{\log }\left(a_{l}\right)+1$.

Proof. of Theorem 2. If $\rho_{\log }(f)=\infty$, then the result is trivial. Next we suppose $\rho_{\log }(f)<\infty$. Recall that we have $\max _{0 \leq j \leq n, j \neq l}\left\{\rho_{\log }\left(a_{j}\right)\right\} \leq \rho_{\log }\left(a_{l}\right)$ and $\sum_{\substack{\log \left(a_{j}\right)=\rho \\ j \neq l}} \tau_{\log }\left(a_{j}\right)<\tau_{\log }\left(a_{l}\right)$. If $\rho_{\log }\left(a_{j}\right)<\rho_{\log }\left(a_{l}\right)$ for all $j=0,1, \cdots, l-$ $1, l+1, \cdots, n$, then Theorem 2 reduces to Theorem 1 . Thus, we assume that at least one of $a_{j}(j=0,1, \cdots, l-1, l+1, \cdots, n)$ satisfies $\rho_{\log }\left(a_{j}\right)=$ $\rho_{\log }\left(a_{l}\right)=\rho$. So, there exists a set $J_{1} \subseteq\{0,1, \cdots, l-1, l+1, \cdots, n\}$ such that for $j \in J_{1}$ we have $\rho_{\log }\left(a_{j}\right)=\rho_{\log }\left(a_{l}\right)=\rho$ and $\sum_{j \in J_{1}} \tau_{\log }\left(a_{j}\right)<\tau_{\log }\left(a_{l}\right)=\tau$ and for $i \in\{0,1, \cdots, l-1, l+1, \cdots, n\} \backslash J_{1}$ we have $\rho_{\log }\left(a_{i}\right)<\rho_{\log }\left(a_{l}\right)=\rho$. Hence, we can choose $\alpha_{1}, \alpha_{2}$ satisfying $\sum_{j \in J_{1}} \tau_{\log }\left(a_{j}\right)<\alpha_{1}<\alpha_{2}<\tau$ such that for any given $\varepsilon\left(0<\varepsilon<\frac{\alpha_{2}-\alpha_{1}}{n}\right)$ and for sufficiently large $r$, we have

$$
T\left(r, a_{j}\right) \leq\left(\tau_{\log }\left(a_{j}\right)+\varepsilon\right)(\log r)^{\rho}, \quad j \in J_{1}
$$

and

$$
T\left(r, a_{i}\right) \leq(\log r)^{\rho_{0}}, \quad i \in\{0,1, \cdots, l-1, l+1, \cdots, n\} \backslash J_{1},
$$

where $1 \leq \rho_{0}<\rho$. By applying Lemma 2 , there exists a subset $E_{2}$ of $[1, \infty)$ that has infinite logarithmic measure such that for all $r \in E_{2}$, we have

$$
T\left(r, a_{l}\right)>\alpha_{2}(\log r)^{\rho} .
$$

By using the assumptions (27), (28), (32), (33) and (34), we obtain from (26) for any given $\varepsilon\left(0<\varepsilon<\min \left\{\frac{\alpha_{2}-\alpha_{1}}{n}, \rho-\sigma\right\}\right)$ and for all $r \in E_{2}$

$$
\begin{aligned}
\alpha_{2}(\log r)^{\rho} \leq & \sum_{j \in J_{1}}\left(\tau_{\log }\left(a_{j}\right)+\varepsilon\right)(\log r)^{\rho}+\sum_{i \in\{0,1, \cdots, l-1, l+1, \cdots, n\} \backslash J_{1}}(\log r)^{\rho_{0}} \\
& +O\left((\log r)^{\rho_{\log }(f)-1+\varepsilon}\right)+(\log r)^{\sigma+\varepsilon} \\
\leq & \left(\alpha_{1}+\varepsilon n\right)(\log r)^{\rho}+n(\log r)^{\rho_{0}}+O\left((\log r)^{\rho_{\log }(f)-1+\varepsilon}\right)+(\log r)^{\sigma+\varepsilon} .
\end{aligned}
$$


It follows that

$$
\begin{aligned}
\left(\alpha_{2}-\alpha_{1}-\varepsilon n\right)(\log r)^{\rho} \leq & n(\log r)^{\rho_{0}} \\
& +O\left((\log r)^{\rho_{\log }(f)-1+\varepsilon}\right)+(\log r)^{\sigma+\varepsilon}
\end{aligned}
$$

Since $0<\varepsilon<\min \left\{\frac{\alpha_{2}-\alpha_{1}}{n}, \rho-\sigma\right\}$, we obtain from (35) that $\rho_{\log }\left(a_{l}\right)=\rho \leq$ $\rho_{\log }(f)-1$.

Proof. of Theorem 3. If $f$ is a non-constant rational function, then $f$ has logarithmic order 1 and $\lambda_{\log }(f)=0$. Thus, the conclusion of the theorem follows immediately. Suppose now that $f$ is transcendental meromorphic function. By (5) we have

$$
\frac{1}{f(z)}=\frac{1}{F}\left(a_{n} \frac{f(z+n)}{f(z)}+\cdots+a_{1} \frac{f(z+1)}{f(z)}+a_{0}\right) .
$$

By (36) and Lemma 5, we have

$$
\begin{aligned}
T(r, f)= & T\left(r, \frac{1}{f}\right)+O(1)=m\left(r, \frac{1}{f}\right)+N\left(r, \frac{1}{f}\right)+O(1) \\
\leq & N\left(r, \frac{1}{f}\right)+m\left(r, \frac{1}{F}\right)+\sum_{j=0}^{n} m\left(r, a_{j}\right) \\
& +\sum_{j=1}^{n} m\left(r, \frac{f(z+j)}{f(z)}\right)+O(1) \\
\leq & N\left(r, \frac{1}{f}\right)+T\left(r, \frac{1}{F}\right)+\sum_{j=0}^{n} T\left(r, a_{j}\right) \\
& +\sum_{j=1}^{n} m\left(r, \frac{f(z+j)}{f(z)}\right)+O(1) \\
\leq & N\left(r, \frac{1}{f}\right)+O\left((\log r)^{\rho_{\log }(f)-1+\varepsilon}\right)+T(r, F)+\sum_{j=0}^{n} T\left(r, a_{j}\right) .
\end{aligned}
$$

Set $\max \left\{\rho_{\log }\left(a_{j}\right)(j=0, \cdots, n), \rho_{\log }(F)\right\}<\rho_{\log }(f)$. Then, by Lemma 3 , there exists a set $E_{3} \subset[1,+\infty)$ with infinite logarithmic measure such that

$$
\max \left\{\frac{T\left(r, a_{j}\right)}{T(r, f)}(j=0, \ldots, n), \frac{T(r, F)}{T(r, f)}\right\} \rightarrow 0, \quad r \rightarrow+\infty, \quad r \in E_{3} .
$$

Thus, by (37) and (38), we have for all $r \in E_{3}, r \rightarrow+\infty$

$$
(1-o(1)) T(r, f) \leq N\left(r, \frac{1}{f}\right)+O\left((\log r)^{\rho_{\log }(f)-1+\varepsilon}\right) .
$$


By (39), we obtain that $\rho_{\log }(f) \leq \lambda_{\log }(f)+1$ and by Lemma 6 , we have $\lambda_{\log }(f)+1 \leq \rho_{\log }(f)$ for every transcendental meromorphic function. Hence, we deduce that $\rho_{\log }(f)=\lambda_{\log }(f)+1$.

Proof. of Theorem 4. Set $w(z)=f(z)-\varphi(z)$. (i) If $\rho_{\log }(f)>$ $\rho_{\log }(\varphi)$, then by Lemma 7 we have $\rho_{\log }(w)=\rho_{\log }(f)$. Substituting $w$ into equation (1), we obtain

$$
\begin{aligned}
& a_{n} w(z+n)+a_{n-1} w(z+n-1)+\cdots+a_{1} w(z+1)+a_{0} w(z) \\
& =-\left(a_{n} \varphi(z+n)+a_{n-1} \varphi(z+n-1)+\cdots+a_{1} \varphi(z+1)+a_{0} \varphi(z)\right) \\
& =A(z) .
\end{aligned}
$$

Since $\varphi$ is not a solution of $(1)$, then $A \not \equiv 0$. By Theorem 2 , we have

$$
\rho_{\log }(f) \geq \max _{0 \leq j \leq n}\left\{\rho_{\log }\left(a_{j}\right)\right\}+1
$$

which implies

$$
\rho_{\log }(w)=\rho_{\log }(f)>\max \left\{\rho_{\log }(A), \rho_{\log }\left(a_{j}\right)(j=0, \cdots, n)\right\} .
$$

Therefore, by Theorem 3 we have $\rho_{\log }(w)=\lambda_{\log }(w)+1$, i.e., $\lambda_{\log }(f-\varphi)+$ $1=\rho_{\log }(f)$

(ii) Suppose now that $\varphi \not \equiv 0$ and $\rho_{\log }(\varphi)<\rho_{\log }\left(a_{l}\right)+1$. Since $\varphi \not \equiv 0$ and $\rho_{\log }(\varphi)<\rho_{\log }\left(a_{l}\right)+1=\max _{0 \leq j \leq n}\left\{\rho_{\log }\left(a_{j}\right)\right\}+1 \leq \rho_{\log }(f)$,

then $A \not \equiv 0$. By (40) and Theorem 3, we obtain $\lambda_{\log }(w)+1=\rho_{\log }(w)$, i.e., $\lambda_{\log }(f-\varphi)+1=\rho_{\log }(f)$. This completes the proof of Theorem 4 .

Proof. of Corollary Setting $g(z)=f(z)-z$. It is clear that $\rho_{\log }(g)=$ $\rho_{\log }(f)$ because $\rho_{\log }(f) \geq \rho_{\log }\left(a_{l}\right)+1>\rho_{\log }(z)=1$. Substituting $f=g+z$ into equation (1), we obtain

$$
\sum_{j=0}^{n} a_{j}(z) g(z+j)=-\sum_{j=0}^{n}(z+j) a_{j}(z) .
$$

In order to prove $\rho_{\log }(f-z)=\lambda_{\log }(f-z)+1$ we need to prove $\sum_{j=0}^{n}(z+j)$ $\times a_{j}(z) \not \equiv 0$. Suppose that $\sum_{j=0}^{n}(z+j) a_{j}(z) \equiv 0$. Then, by the conditions (3), (4) and Lemma 8 we have

$$
\rho_{\log }(0)=\rho_{\log }\left(\sum_{j=0}^{n}(z+j) a_{j}(z)\right)=\rho_{\log }\left(a_{l}\right) \geq 1
$$


which is a contradiction. Hence, by applying Theorem 4 we obtain

$$
\lambda_{\log }(f-z)+1=\rho_{\log }(f-z)=\rho_{\log }(f) .
$$

Acknowledgements. The author is grateful to the referees for their valuable comments and suggestions which lead to the improvement of this paper. This paper is supported by University of Mostaganem (UMAB) (CNEPRU Project Code B02220120024).

\section{References}

[1] CAO T.B., Liu K., Wang J., On the growth of solutions of complex differential equations with entire coefficients of finite logarithmic order, Math. Reports 15(65), 3(2013), 249-269.

[2] Chern Peter T.Y., On meromorphic functions with finite logarithmic order, Trans. Amer. Math. Soc., 358(2)(2006), 473-489.

[3] Chiang Y.M., Feng S.J., On the Nevanlinna characteristic of $f(z+\eta)$ and difference equations in the complex plane, Ramanujan J., 16(1)(2008), 105-129.

[4] Halburd R.G., Korhonen R.J., Difference analogue of the lemma on the logarithmic derivative with applications to difference equations, J. Math. Anal. Appl., 314(2)(2006), 477-487.

[5] Halburd R.G., Korhonen R.J., Nevanlinna theory for the difference operator, Ann. Acad. Sci. Fenn. Math., 31(2)(2006), 463-478.

[6] Hayman W.K., Meromorphic functions, Oxford Mathematical Monographs Clarendon Press, Oxford 1964.

[7] Heittokangas J., Wen Z.T., Functions of finite logarithmic order in the unit disc, Part I. J. Math. Anal. Appl., 415(1)(2014), 435-461.

[8] Heittokangas J., Wen Z.T., Functions of finite logarithmic order in the unit disc, Part II. Comput. Methods Funct. Theory, 2014, DOI 10.1007/s40315-014-0089-4.

[9] Laine I., Yang C.C., Clunie theorems for difference and $q$-difference polynomials, J. Lond. Math. Soc. (2), 76(3)(2007), 556-566.

[10] Latreuch Z., BelaïDi B., Growth and oscillation of meromorphic solutions of linear difference equations, Mat. Vesnik, 66(2)(2014), 213-222.

[11] Wen Z.T., Finite logarithmic order solutions of linear $q$-difference equations, Bull. Korean Math. Soc., 51(1)(2014), 83-98.

[12] Yang C.C., Yi H.X., Uniqueness Theory of Meromorphic Functions, Applications, 557. Kluwer Academic Publishers Group, Dordrecht, 2003.

$$
\begin{gathered}
\text { Benharrat Belaïdi } \\
\text { Department OF Mathematics } \\
\text { Laboratory OF Pure and ApPlied Mathematics } \\
\text { University OF Mostaganem (UMAB) } \\
\text { B. P. } 227 \text { Mostaganem-(Algeria) } \\
\text { e-mail: } \text { belaidi@univ-mosta.dz }
\end{gathered}
$$

Received on 05.08.2014 and, in revised form, on 10.04.2015. 